\title{
Changes in osteoblast and osteoclast cell count after moringa oleifera leaf extract administration during orthodontic tooth movement
}

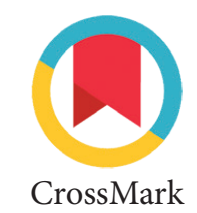

Rika D. Syarif, ${ }^{1 *}$ Tuti Kusumaningsih, ${ }^{2}$ Ira Arundina ${ }^{2}$

\section{Abstract}

Objective: The aim of this study was to analyze the effect of moringa leaf extract administration at $5 \%, 10 \%$, and $20 \%$ concentration toward osteoblast and osteoclast cell count in tension area of Cavia cobaya tooth movement.

Material and Methods: This study was an experimental research with post test only control group design conducted on Cavia Cobaya. The samples were divided into four groups, namely control group (given orthodontic forces), P1 group (given orthodontic forces and moringa oleifera extract at 5\% concentration), P2 group (given orthodontic forces and moringa oleifera extract at $10 \%$ concentration) and $\mathrm{P} 3$ group (given orthodontic forces and moringa oleifera extract at $20 \%$ concentration). The samples were sacrificed in day 15 and the tissue were collected for histological test to examine osteoblast and osteoclast cell count.

Results: The Anova test showed a significant difference of osteoblast and osteoclast cell count among groups $(p<0.05)$.

Conclusion: Moringa leaves extract administration at 20\% concentration for seven days shows higher number of osteoblast and lower number of osteoclast compared to control group, 5\% and 10\% extract group.
'Department of Orthodontic, Faculty of Dentistry, Hasanuddin University, Makassar, Indonesia. ${ }^{2}$ Department of Oral Biology, Faculty of Dental Medicine, Universitas Airlangga, Surabaya, Indonesia.

*Correspondence to: Rika D. Syarif, Department of Orthodontic, Faculty of Dentistry, Hasanuddin University, Makassar, Indonesia. rik.damayanti17@gmail.com

Received: 5 June 2020

Revised: 14 June 2020

Accepted: 16 July 2020

Available Online: 1 August 2020
Keywords: Moringa oleifera, Orthodontic tooth movement, Osteoblast, 0steoclast, Osteogenesis

Cite this Article: Syarif, R.D., Kusumaningsih, T., Arundina, I. 2020. Changes in osteoblast and osteoclast cell count after moringa oleifera leaf extract administration during orthodontic tooth movement. Journal of Dentomaxillofacial Science 5(2): 98-102. D0I: 10.15562/jdmfs.v5i2.1081

\section{Introduction}

The application of orthodontic force lead to tooth movement, indicated by changes in periodontal ligament and alveolar bone which further activate alveolar bone remodeling. ${ }^{1,2}$ Bone remodeling denotes active and dynamic process involving bone cell interaction, systemic hormones, cytokine and growth factor, aiming to maintain bone resorption by osteoclast and bone formation by osteoblast in balance. ${ }^{3,4}$ In orthodontic tooth movement, the bone formation process is more time-consuming than bone resorption. ${ }^{5}$ The bone resorption process takes place in about 4-6 weeks, while the bone formation process required 2 months in every remodeling cycle. A new bone formation in tension area is essential to maintain the long-term stability of the moved tooth. ${ }^{1}$

The success of orthodontic treatment rest on the outcome of remodeling process, which is determined by the quantity and quality of osteoblast and osteoclast. ${ }^{3}$ Osteoblast and osteoclast constitutes the two important cells involved in orthodontic tooth movement. Osteoblast denotes the main cell in bone tissue and bone formation, which is essential for bone morphology and the stability of moved tooth. Osteoblast regulate osteoclast recruitment and activity as well as control the phase of bone formation and resorption in bone remodeling cycle., ${ }^{1,2}$ A continuous mechanical force during orthodontic treatment may increase the function of mature osteoblast, along with the increase in bone formation and osteoblast precursor's differentiation. Osteoclast denotes a giant cell with multiple nucleus which degrade and resorb the bone. Osteoclast collaborate with osteoblast in bone remodeling process. ${ }^{2,3}$

Moringa oleifera leaves evidently contain various natural anti-inflammation and antioxidant substances namely flavonoid, phenolic and carotenoid. Besides, moringa leaves also rich in protein, vitamin $\mathrm{C}$, vitamin $\mathrm{A}$, calcium and potassium. ${ }^{6}$ Flavonoid may act as antioxidant by scavenging free radical and protecting the cell from oxidative stress. ${ }^{7}$ Based on previous study, flavonoid can stimulate osteoblast proliferation and differentiation. ${ }^{8}$ Other study by Marupanthorn proved that ethanol extract of moringa leaves could increase osteogenic differentiation of porcine bone marrow derived mesenchymal stem cells (MSCs) by increasing osteoblast proliferation and differentiation. Against the foregoing background, this study is aiming to investigate the effect of moringa leaves extract administration toward osteoblast and osteoclast cell count during orthodontic tooth movement. 


\section{Material and Methods}

This was a laboratory based experimental study with post test only control group design. Identification and preparation of moringa oleifera leaves extract were performed in Pharmacognosy Laboratory, Faculty of Pharmacy, Hasanuddin University. The animal treatments were conducted in Biochemistry Laboratory, Faculty of Medicine Universitas Airlangga. All the procedures in this study have received approval of Institutional Ethical Committee of Faculty of Dentistry Universitas Airlangga.

Twenty eight male, 3-4 months old cavia cobaya used as samples. The inclusion criteria were physically healthy, have complete incisors, and have a good oral and periodontal health. After being acclimatized for seven days, Cavia cobaya were randomly divided into 4 groups; control group (K) were given orthodontic forces, $\mathrm{P} 1$ group were given orthodontic forces and 5\% moringa oleifera extract, P2 group were given orthodontic forces and 10\% moringa oleifera extract, and P3 were given orthodontic forces and $20 \%$ moringa oleifera extract.

Orthodontic force was applied in the mesial side of both upper insisors of cavia cobaya using prefabricated niti open coil spring and measured with tension gauge. The moringa oleifera extract were processed by maceration method using $70 \%$ ethanol. The extract was diluted with $1 \%$ CMC $\mathrm{Na}$ gel to form moringa oleifera extract 5\%, 10\% and $20 \%$ concentration. The aplication of moringa oleifera extract was done on day 8 after orthodontic force was applied, for 7 days. The moringa oleifera extract were administered using tuberculin syringe at $0.05 \mathrm{ml}$.

On day 15 , cavia cobaya were sacrificed and the alveolar bones were dissected. The acquired alveolar bones were then decalcified using ethylene diamine tetra acetic acid (EDTA), for 30 days and made into paraffin blocks subsequently. The examination of osteoblast and osteoclast cell count were performed using a light microscope at $400 \times$ magnification.

The collected data were analyzed with using one-way ANOVA to identify differences among groups with significance value of 0.05 .

\section{Results}

A histological examination using Hematoxylin Eosin (HE) staining was carried out on the number of osteoblast and osteoclast in tension area of each

Table 1 Mean and standard deviation of osteoblast and osteoclast cell count of each group

\begin{tabular}{lllll}
\hline Variable & Treatment Group & N & Mean \pm SD & p \\
\hline Osteoblast cell count & Control (K) & 7 & $10.43 \pm 2.07$ & $0.000^{*}$ \\
& Extract 5\% (P1) & 7 & $13.86 \pm 2.27$ & \\
& Extract 10\% (P2) & 7 & $21.29 \pm 2.29$ & \\
& Extract 20\% (P3) & 7 & $25.29 \pm 0.49$ & $0.000^{*}$ \\
Osteoclast cell count & Control (K) & 7 & $22.14 \pm 4.02$ & $16.86 \pm 2.73$ \\
& Extract 5\% (P1) & 7 & $11.71 \pm 1.98$ & \\
& Extract 10\% (P2) & 7 & $8.71 \pm 1.25$ & \\
\hline
\end{tabular}

${ }^{*}$ Level of Significance $\mathrm{p}<0.05$

Table 2 LSD test of osteoblast cell count following moringa oleifera extract administration

\begin{tabular}{lcccc}
\hline Treatment Group & Control (K) & Extract 5\% (P1) & Extract 10\% (P2) & Extract 20\% (P3) \\
\hline Control (K) & - & $0.003^{\star}$ & $0.000^{*}$ & $0.000^{\star}$ \\
Extract 5\% (P1) & - & - & $0.000^{*}$ & $0.000^{*}$ \\
Extract 10\% (P2) & - & - & - & $0.001^{*}$ \\
\hline
\end{tabular}

${ }^{\star}$ Level of Significance $\mathrm{p}<0.05$

Table 3 LSD test of osteoclast cell count after MO extract administration

\begin{tabular}{lcccc}
\hline Treatment Group & Control (K) & Extract 5\% (P1) & Extract 10\% (P2) & Extract 20\% (P3) \\
\hline Control (K) & - & $0.001^{*}$ & $0.000^{*}$ & $0.000^{*}$ \\
Extract 5\% (P1) & - & - & $0.002^{*}$ & $0.000^{*}$ \\
Extract 10\% (P2) & - & - & - & $0.048^{*}$ \\
\hline
\end{tabular}

${ }^{\star}$ Level of Significance $\mathrm{p}<0.05$ 
groups. The result was shown in figure 1 and figure 2 respectively. The mean and standard deviation of osteoblast and osteoclast cell count in tension area of each groups after moringa oleifera extract administration were tabulated and presented in table 1 . According to the observation, the treatment groups showed a higher number of osteoblast compared to the control group. The highest mean of osteoblast number recorded was from P3 group $(25.29 \pm 0.49)$ and the lowest was P1 group (13.86 \pm 2.27$)$. As for osteoclast cell count, the cell count of treatment groups was lower than the control groups. The highest osteoclast cell count recorded was from P1
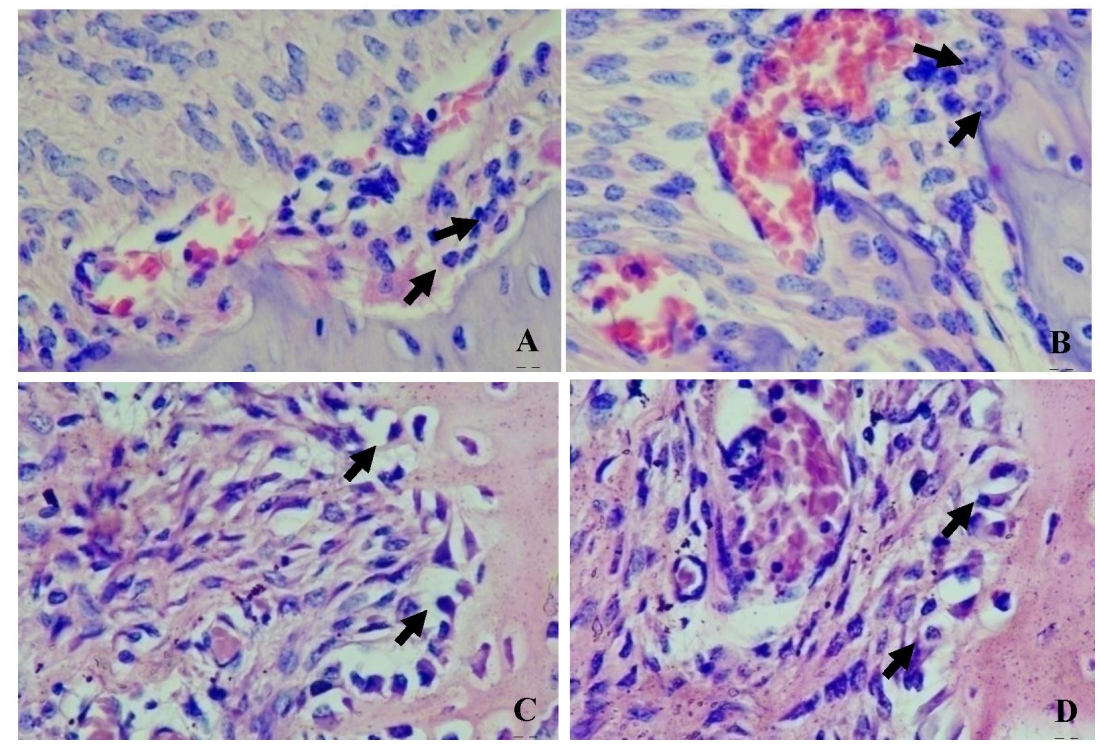

Figure 1 Histological features of osteoblas on tension side (black arrow). A. Control group, B. 5\% extract group, C. 10\% extract group, D. $20 \%$ extract group. (HE staining, magnification $400 \times$ )
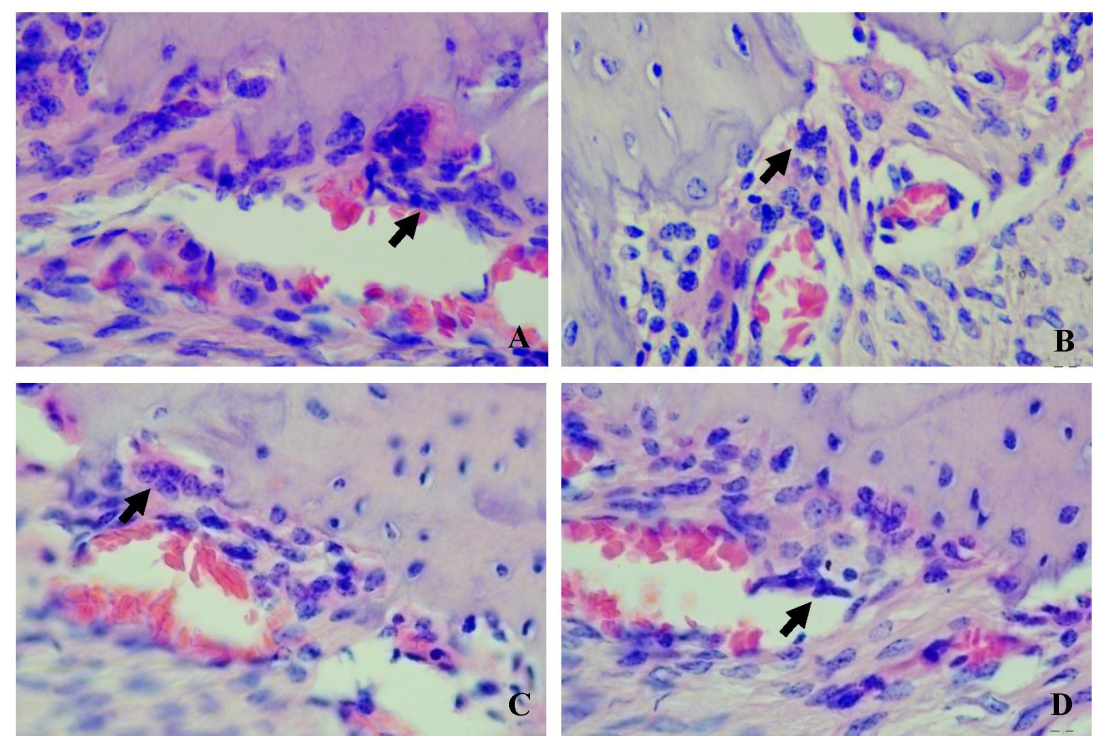

Figure 2 Histopathologic features of Osteoclast on tension side (black arrow). A. Control group, B. 5\% extract group, C. $10 \%$ extract group, D. 20\% extract group. (HE staining, magnification $400 \times$ ) group $(16.86 \pm 2.73)$ and the lowest was P3 group $(8.71 \pm 1.25)$.

The initial Kolmogorov-smirnov test was performed and indicated that the acquired data of osteoblast cell count were normally distributed ( $\mathrm{p}=0.534$ ), then further levene test was performed, showing that the data were also homogeny $(\mathrm{p}=0.147)$. The data were analyzed using One-way ANOVA. There was a significant difference of osteoblast between the four groups $(\mathrm{p}=0.000)$ table 1 . The osteoblast cell count was further analyzed using Post Hoc LSD test, which are shown in table 2. The LSD test revealed that the osteoblast cell count of P3 group were significantly higher compared to P2 group ( $\mathrm{p}=0.001), \mathrm{P} 1$ group $(\mathrm{p}=0.000)$, and control group $(\mathrm{p}=0.000)$. The result of $\mathrm{P} 2$ group were also significantly higher than $\mathrm{P} 1(\mathrm{p}=0.000)$ and control group ( $\mathrm{p}=0.000$ ), and lastly the osteoblast cell count of P1 group were significantly higher compared to the control group ( $\mathrm{p}=0.003)$.

Kolmogorov-smirnov and levene test also indicated that the osteoclast cell count was normally distributed $(\mathrm{p}=0.643)$ and homogeny $(\mathrm{p}=0.090)$. A difference test was also performed, showing a significant difference among groups $(\mathrm{p}=0.000)$. The data were subsequently analyzed using post hoc LSD test, and the result is tabulated in table 3.

LSD test showed that the osteoclast cell count of P3 group was considerably lower compared to those of the $\mathrm{P} 2$ group $(\mathrm{p}=0.048), \mathrm{P} 1$ group $(\mathrm{p}=0.000)$, and control group $(\mathrm{p}=0.000)$. The least osteoclast cell count recorded was $\mathrm{P} 3$, which followed by $\mathrm{P} 2$ group that significantly lower than P1 group $(\mathrm{p}=0.002)$ and control group $(\mathrm{p}=0.000)$. Finally, the osteoblast cell count of P1 group was significantly lower compared to the control group ( $\mathrm{p}=0.001)$.

\section{Discussion}

This research is aiming to analyze the osteogenic activity after moringa oleifera extract administration in tension area of orthodontic tooth movement. Tooth movement may cause several physiological changes in periodontal tissue, which further affect the activity, metabolism, and communication of cells that involved in the process, as a response to the orthodontic forces. ${ }^{10}$ Nitric Oxide denotes the key regulator of the bone response toward mechanical forces, which has been proven its contribution in bone formation, osteoclast activity, also preventing apoptosis of osteocyte and osteoblast. ${ }^{5}$ A large amount of Nitric Oxide may inhibit and reduce osteoblast activity and differentiation, and also remineralisazation and osteogenesis process. ${ }^{11}$

Moringa leaves (moringa oleifera) constitutes a resource of polyphenol such as flavonoid, which 
possess a high anti-oxidant and anti-inflammation characteristics. The main flavonoid detected in moringa leaves is myrecytin, quercetin and kaempferol. ${ }^{12}$ The anti-oxidant characteristics possessed by polyphenol may inhibit reactive oxygen species (ROS), also maintain the osteoblast and osteocyte vitality, which play role in osteoblast activity and osteogenesis, and reduce osteoclast differentiation and activity. ${ }^{11,13}$ Through the anti-oxidant mechanism, polyphenol has been proven to reduce the undesirable effect of oxidative stress in osteoblast, by means of tumor necrosis- $\alpha$ (TNF- $\alpha$ ) inhibition so that apoptosis will not occur. ${ }^{14}$

This study revealed that the administration of moringa oleifera extract at 5\%,10\% and $20 \%$ concentration were able to increase the osteoblast cell count. However, the highest osteoblast cell count recorded was of the treatment group which was administered moringa oleifera extract at $20 \%$ concentration. This possibly due to the phytoestrogen contained in moringa leaves. Estrogen affect bone anabolism by increasing the osteoblast function and inhibiting the osteoclastogenesis. The act of estrogen mainly mediated by the high affinity of nuclear receptor. Moreover, some other flavonoids are considered fitoestrogen due to its ability to bind and give signal trough the estrogen receptor. ${ }^{15}$ This result is in accordance to the previous study which indicated that a certain flavonoid, namely qurcetin and rutin may increase the proliferation and differentiation of osteoblast, by means of increasing the stimulus to produce osteoprogenitor. ${ }^{16}$

That phytoestrogen can increase MSCs differentiation and inhibit adipogenic differentiation through non-genomic ER-mediated mechanism. Other in vitro and in vivo studies also showed that flavonols, quercetin and kaempferol constitute bioactive molecules which can prevent bone loss due to low level of estrogen during menopause. Besides, quercetin and kaempferol are also able to prevent osteopenia in ovariectomy rat model. ${ }^{17}$

This study also showed that the administration of moringa oleifera extract at 5\%, $10 \%$, and $20 \%$ concentration could significantly reduce the osteoclast cell count, compared to the control group. However, the lowest osteoclast cell count recorded was from group administered with moringa oleifera extract at $20 \%$ concentration. The result corresponds to the previous study which proved that kaempferol and quercetin were able to inhibit bone resorption and increase mature osteoclast apoptosis in rabbit ${ }^{15}$ while other study by Wattel et al. ${ }^{18}$ also stated that quercetin in low concentration could inhibit osteoclast differentiation, induce mature osteoclast differentiation, inhibit bone resorption by impairing the regulation of NF- $\kappa \mathrm{B}$ and activation of transcription AP-1 in osteoclast precursor.

The flavonoid contained in moringa leaves have a high osteogenic activity, which may increase osteoblastic activity. Flavonoid can manage cell function by stimulating TGF- $\beta$ production, whereas TGF- $\beta$ induces proliferation and migration of osteoblast. ${ }^{19}$ The increase of osteoblastic proliferation and activation may increase the amount of osteoblast, which further increase the expression of osteoprotegerin (OPG). ${ }^{4}$ An increase of OPG is very influential to the osteoclast activation, since OPG is the decoy receptor for Receptor Activator of Nuclear Factor Kappa-B Ligand (RANKL), by inhibiting the interaction between RANKL and Receptor Activator of Nuclear Factor Kappa-B (RANK) in precursor osteoclast cell membrane, thus inhibit the osteoclastogenesis. The inhibited osteoclastogenesis may reduce the rate and intensity of osteoclastogenesis and lead to osteoclast apoptosis, thus reduce the bone resorption and increase the bone formation. ${ }^{20}$

\section{Conclusion}

Administration of moringa oleifera extract at 5\%, $10 \%$, and $20 \%$ concentration can increase the number of osteoblast and decrease the number of osteoclast in tension area in orthodontic tooth movement of cavia cobaya. Thus, the use of moringa leaves as an alternative to accelerate bone formation, especially in orthodontic treatment can be considered.

\section{Acknowledgment}

We would like to appreciate Faculty of Dentistry Hasanuddin University and Airlangga University for their support to this study.

\section{Conflict of Interest}

The authors report no conflict of interest.

\section{References}

1. Mao Y, Wang L, Zhu Y, et al. Tension force-induced bone formation in orthodontic tooth movement via modulation of the GSK-3 $\beta / \beta$-catenin signaling pathway. J Mol Histol 2018;49:75-84

2. Nagarajan D, Usha K, Rao GU, et al. Biomarkers in orthodontics. Int J Oral Heal Med Res 2015;2: 88-90.

3. Ariffin SHZ, Yamamoto Z, Abidin IZZ, et al. Cellular and molecular changes in orthodontic tooth movement. Sci World J 2011;11: 1788-1803.

4. Rucci N. Molecular biology of bone remodelling. Clin Cases Miner Bone Metab 2008;5: 49-56.

5. Baloul SS. Osteoclastogenesis and osteogenesis during tooth movement. Front Oral Biol 2016;18: 75-79. 
6. Dubey DK, Dora J, Kumar A, et al. A multipurpose treemoringa oleifera. J Pharm Chem Sci 2013;2: 415-423.

7. Yokomizo A, Moriwaki M. Effects of uptake of flavonoids on oxidative stress induced by hydrogen peroxide in human intestinal caco-2 cells. Biosci, Biotechnol, Biochem 2006;70: 1317-1324.

8. Zhang DW, Cheng Y, Wang NL, et al. Effects of total flavonoids and flavonol glycosides from epimedium koreanum nakai on the proliferation and differentiation of primary osteoblasts. Phytomedicine 2008;15: 55-61.

9. Marupanthorn K, Kedpanyapong W. The effects of moringa oleifera lam. leaves extract on osteogenic differentiation of porcine bone marrow derived mesenchymal stem cells. Int Conf Adv Agric Biol Ecol Sci 2016: 29-32.

10. Garlet TP, Coelho U, Repeke CE, et al. Differential expression of osteoblast and osteoclast chemmoatractants in compression and tension sides during orthodontic movement. Cytokine 2008;42: 330-335.

11. Domazetovic V. Oxidative Stress in bone remodeling: role of antioxidants. Clin Cases Miner Bone Metab 2017;14: 209-216.

12. Vergara-Jimenez $M$, Almatrafi MM, Fernandez $M$. Bioactive components in moringa oleifera leaves protect against chronic disease. Antioxidants 2017;6: 1-13.

13. Dudaric L, Fuzinac-Smojver A, Muhvic D, et al. The role of polyphenols on bone metabolism in osteoporosis. Food Res Int 2015;77: 290-298.

14. Torre E. Molecular signaling mechanisms behind polyphenol-induced bone anabolism. Phytochem Rev 2017;16: 1183-1226.
15. Pang JL, RicuperoDA, Huang S, et al. Differential activity of kaempferol and quercetin inattenuating tumor necrosis factor receptor familysignaling in bone cells. Biochemical Pharmacology 2006;71: 818-826.

16. Srivastava S, Bankar R, Roy P. Assessment of the role of flavonoids for inducing osteoblast differentiation in isolated mouse bone marrow derived mesenchymal stem cells. Phytomedicine 2013;20: 683-690.

17. Horcajada M-N, Offord E. Naturally plant-derived compounds: role in bone anabolism. Curr Mol Pharmacol 2012;5: 205-218.

18. Wattel A, Kamel S, Prouillet C, et al. Flavonoid Quercetin decreases osteoclastic differentiation induced by RANKL via a mechanism involving NFKB and AP-1. J Cell Biochem 2004;92: 285-295.

19. Ismardianita E, Nasrul E, Yanwirasti, et al. Effect of ethanol extract of myrmecodia pendens on TGF- $\beta 1$ expression and osteoblast cells after tooth extraction (experimental research on cavia cobaya). J Dentomaxillofac Sci 2017;2: 150-154.

20. Kajiya M, Giro G, Taubman MA, et al. Role of Periodontal Pathogenic Bacteria in RANKL-mediated Bone Destruction in Periodontal Disease. J Oral Microbiol 2010;2:1-17.

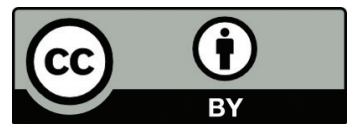

This work is licensed under a Creative Commons Attribution 